\title{
Anabases
}

ANABASES Traditions et réceptions de l'Antiquité

$17 \mid 2013$

Varia

\section{Marine MOLINS, Charles Fontaine traducteur. Le poète et ses mécènes à la Renaissance}

\section{Pascale Chiron}

\section{OpenEdition}

\section{Journals}

Édition électronique

URL : http://journals.openedition.org/anabases/4285

DOI : $10.4000 /$ anabases.4285

ISSN : 2256-9421

\section{Éditeur}

E.R.A.S.M.E.

\section{Édition imprimée}

Date de publication : 1 mars 2013

Pagination : 295-298

ISSN : 1774-4296

\section{Référence électronique}

Pascale Chiron, « Marine mouıns, Charles Fontaine traducteur. Le poète et ses mécènes à la Renaissance », Anabases [En ligne], 17 | 2013, mis en ligne le 01 avril 2013, consulté le 22 septembre 2020. URL: http://journals.openedition.org/anabases/4285 ; DOI : https://doi.org/10.4000/anabases.4285

Ce document a été généré automatiquement le 22 septembre 2020.

(c) Anabases 


\title{
Marine MOLINS, Charles Fontaine traducteur. Le poète et ses mécènes à la Renaissance
}

\author{
Pascale Chiron
}

\section{RÉFÉRENCE}

Marine MoLins, Charles Fontaine traducteur. Le poète et ses mécènes à la Renaissance, Genève, Droz, 2011, $367 \mathrm{p}$.

78,25 euros / 978-2-600-01509-7.

1 Jusqu'à ces dernières années, Charles Fontaine n'avait pas suscité l'intérêt des chercheurs et semblait définitivement relégué au rang des minores dans l'ombre de Clément Marot. Seule une monographie anglaise de R. L. Hawkins (Maistre Charles Fontaine Parisien, 1916) et quelques travaux (ceux de Grace Frank ou de Raffaele Scalamandre) avaient éclairé le travail de cet érudit de la Renaissance, à la fois poète, humaniste, traducteur, éditeur. Mais le vent tourne et le travail de Marine Molins s'inscrit dans ce renouveau de l'intérêt des chercheurs pour Charles Fontaine, qu'un colloque à Lyon en juin 2009, organisé par E. Rajchenbach-Teller et G. de Sauza («Charles Fontaine : un humaniste parisien à Lyon») a définitivement exhumé, le tirant d'une place négligeable qu'il ne méritait pas.

Dans son livre Charles Fontaine traducteur, Marine Molins fait un choix original, qui se situe dans la suite de son travail de thèse (Traduction et narration à la Renaissance), puisqu'elle s'intéresse à une partie du corpus du poète qui n'avait encore jamais fait l'objet d'un travail global: les traductions. L'hypothèse qu'elle vérifie ici de manière convaincante est que ces traductions ont un lien étroit avec leurs dédicataires, soit que le choix de tel ou tel texte-source reflète le goût d'un mécène ou d'un dédicataire auquel on veut plaire, soit que la façon même de traduire ou de commenter témoigne 
d'une adaptation au goût ou aux besoins des dédicataires, et parfois même d'une volonté de former ce goût.

3 Ce choix d'étudier le lien entre le traducteur et ses dédicataires ou mécènes explique que le livre progresse selon un axe chronologique qui nous permet de resituer charles Fontaine dans une histoire à la fois princière, éditoriale et poétique. Il permet aussi de restituer au travail de traduction une dimension sociale et politique essentielle pour comprendre la Renaissance des Lettres au Xvie siècle. Du point de vue de l'histoire du livre, Marine Molins nous permet de mesurer combien le manuscrit reste très présent dans ce siècle où se développe l'imprimerie. Les commentaires de Fontaine dans ses traductions laissent penser d'ailleurs à deux types de réception, ciblée ou plus anonyme, correspondant à ces deux manières de diffuser une traduction au XVI $\mathrm{e}^{\mathrm{e}}$ siècle.

4 À tout moment, Charles Fontaine semble faire preuve d'une grande lucidité dans ses traductions: il ne cesse de réfléchir sur ses choix. Il est mû par le souci d'une traduction exacte dont on a peu d'exemples avant la fin du $\mathrm{xv}^{\mathrm{e}}$ siècle, mais aussi par la conscience que la traduction doit tenir compte de la spécificité de chaque langue. Marine Molins, qui rapporte l'idée de «destruction» (p. 24) du texte originel, terme trop fort à notre avis, montre finalement de manière plus nuancée les tensions qui existent entre la nécessité de traduire au plus près du texte et le désir d'illustrer la langue française et donc de ne pas la dénaturer par une expression trop littérale qui lui ôterait toute poésie. La traduction devra chercher à restituer, certes, la « robe » du texte (les mots), mais surtout le « corps » du texte (son sens) et sa "grâce naturelle » (l'âme), comme Fontaine le rappelle dans sa préface aux Remèdes d'Amour paru en 1555.

5 Le début de la carrière de Fontaine est lié à la politique de François I ${ }^{\mathrm{er}}$, qui favorise la redécouverte des textes anciens et leurs traductions, et à la fréquentation de poètes (qui sont aussi parfois valets de chambre à la cour) comme Marot, Héroët, Salel, Bonaventure Des Périers ou Antoine Du Moulin, bien introduits auprès du roi ou de Marguerite de Navarre. Marine Molins montre ainsi que la traduction intégrale et en vers de trois épîtres de saint Paul s'inscrit dans un intérêt que partagent la sœur du roi et les membres du cercle de Meaux pour l'auteur des épîtres, saint Paul, et la traduction en français de textes bibliques. Charles Fontaine s'appuie d'ailleurs sur deux versions des épîtres, celle de la Vulgate et celle de Jacques Lefèvre d'Étaples, membre du cercle de Meaux, preuve à la fois de sa rigueur philologique et de cette volonté de se rapprocher du cercle évangélique.

6 C'est en faisant partie de l'entourage de Jean Brinon, grâce à Louis Chesneau, que Charles Fontaine se met à traduire des œuvres profanes comme les élégies d'Ovide et Catulle. Il adressera également à ce mécène sa traduction en décasyllabes des Remèdes d'Amour. Marine Molins montre par des analyses de détails très bien choisies combien le travail de Fontaine, malgré sa fidélité avérée à l'original, peut infléchir ses traductions vers plus d'érotisme, de sensualité, voire d'attendrissement, pour s'adapter peut-être plus sûrement à l'état d'âme du dédicataire, qui venait de subir quelques déconvenues amoureuses.

7 Dans ce même souci de recherche de protecteur et d'adaptation de ses traductions, Fontaine accompagne le maréchal et conseiller très proche de François $\mathrm{I}^{\mathrm{er}}$, Claude d'Annebault, en Italie et traduit pour lui le Translat de duel, peut-être tiré du De duello d'Alciat; mais le manuscrit de Fontaine est aujourd'hui perdu, comme sa traduction d'une Chiromance qu'il aurait renoncé à dédier au maréchal. En revanche, sa traduction en prose du premier livre d'Artémidore sur l'interprétation des songes nous est 
conservée : elle paraît à Lyon en 1546 chez le grand éditeur Jean de Tournes, elle sera augmentée de deux autres livres en 1555. La préface et les commentaires mettent en valeur l'actualité des débats sur le songe qui font apparaître le traducteur comme un homme de son temps. Pour ce qu'il a gardé du texte-source d'Artémidore, Fontaine se montre par ailleurs fidèle à l'original, mais il produit en fait un épitomé : il fait disparaître des chapitres ou des passages entiers où il est question des dieux païens. Marine Molins avance l'hypothèse que le traducteur a cherché ainsi à s'adapter au caractère austère et pieux du maréchal.

Jusque vers 1550, l'activité de traduction de Fontaine semble donc étroitement dépendante de ses commanditaires ou dédicataires (voir également les traductions du De Asse ou des Vies des douze Césars adressées à Monsieur d'Ivor, secrétaire du roi), même si ses intérêts d'humaniste pour des débats d'actualité et ses scrupules d'exactitude dans la traduction restent manifestes, signes d'un engagement personnel.

Mais c'est avec sa traduction des Héroïdes, dédiée à la famille de Crussol (auprès de qui Pierre Saliat l'a introduit), que Fontaine semble véritablement faire preuve de maturité et d'indépendance dans ses choix. Marine Molins consacre l'essentiel de son étude à cette traduction dont les épîtres liminaires, les préfaces, annotations et avis du traducteur au lecteur méritaient en effet une attention particulière puisque Fontaine commente ses difficultés et ses choix personnels de traducteur. Ses commentaires des Héroïdes montrent qu'il s'appuie, en bon humaniste philologue, sur plusieurs éditions latines qu'il confronte, dont celle de G. Morillon à laquelle il reprendra quelques « arguments ».

L'étude de Marine Molins replace plus globalement la traduction de Fontaine dans l'histoire de la réception en France des Héroïdes, depuis le Moyen Âge en passant par la traduction d'Octovien de Saint-Gelais et jusqu'à celle de Du Bellay, dont la traduction de l'héroïde de Didon est contemporaine de celle de Fontaine. Marine Molins retrace scrupuleusement l'histoire de l'édition de ces traductions en confrontant les choix métriques, strophiques, poétiques des traducteurs pour dégager la spécificité de chacun et la façon dont chacun cherche à illustrer la langue française : Fontaine a en effet profité de ce déplacement des Héroïdes du milieu scolaire où elles étaient étudiées vers l'univers poétique pour entrer en compétition avec ses contemporains, et cela sans aucune agressivité, simplement avec la conscience de faire partie d'un mouvement commun destiné à faire progresser la langue française. Ainsi l'édition de Fontaine de 1552 inclura-t-elle des "Contrepistres » de Michel d'Amboise, supprimées en 1556, tandis que sont ajoutées des épîtres d'Octovien de Saint-Gelais que Fontaine lui-même n'avait pas traduites et d'autres dues à Saint-Romard, en plus d'autres textes en rapport avec les Héroïdes. L'édition est enrichie de bois gravés attribués à Jean Cousin.

11 Que cette traduction ait été une commande ou qu'elle ait simplement rencontré les goûts de ses destinataires, nous avons en tout cas une preuve de son succès dans le fait que des plaques émaillées datées de 1564 reprendront les noms francisés de la traduction de Fontaine. Les Héroïdes ont rencontré un public marqué par les guerres d'Italie qui éloignaient les grands de la cour, et auquel le thème de la séparation et de l'absence n'était donc pas étranger. Mais elles ont rencontré aussi le goût pour le genre épistolaire et élégiaque caractéristique de cette Renaissance comme le rappelle Marine Molins (dont l'analyse de l'épître de Maguelonne de Marot pourrait être à cet égard plus convaincante, p.115). Fontaine explique son choix par l'utilité et le plaisir que 
procurent ces lettres, mais aussi par la brièveté et la "vérité historique » qui les caractérisent et les font préférer aux Métamorphoses.

La dernière partie de l'ouvrage de Marine Molins présente les dernières traductions de Fontaine : celles d'ouvrages gnomiques adressés aux enfants d'Henri II qui paraissent à Lyon entre 1557 et 1558 (les Dits des sept sages et les Sentences, les Mimes de Publilius Syrus, première traduction en français de cette œuvre, et les Distiques de Caton). L'utilité morale se combine ici, comme pour les Héroïdes, au goût de la cour pour ce genre de littérature. Charles Fontaine traduit et commente ces textes, et Marine Molins nous convainc de la capacité du traducteur à ajouter une touche personnelle et originale à son travail tout en se glissant dans un genre à la mode. Visée savante et mondaine, alliée à l'originalité, c'est ce dont témoigne également le dernier texte analysé par Marine Molins, les Énigmes, que Fontaine traduit de Symposius.

C'est donc un ouvrage extrêmement utile et nouveau que nous propose Marine Molins. Si quelques maladresses, souvent d'expression (exemple: « textes de l'Antiquité et des autres pays", p. 45), émaillent ici ou là le texte, l'effet produit par cette étude est indéniable : Charles Fontaine apparaît comme un auteur bien plus que "marotique ", un auteur qui aura saisi toutes les modes, tous les goûts qui peuvent à la fois rencontrer les siens et lui permettre de s'assurer la protection des plus grands. Ce faisant, parallèlement à cette variété à la fois dans les textes traduits et les protecteurs recherchés, apparaît l'unité d'un travail qui confronte sans cesse l'idéal d'une traduction exacte et l'expérience du traducteur qui l'oblige à penser la traduction comme une nouvelle création, au plus grand bénéfice de la langue française.

Marine Molins parvient donc à déplacer à la fois l'image jusque-là peu valorisée de l'auteur Charles Fontaine et celle de l'exercice de traduction. Par ailleurs, elle reproduit en annexe un ensemble de textes précieux pour le lecteur: la traduction des trois épîtres de saint Paul, qui constitue un inédit, la traduction d'élégies d'Ovide et de Catulle, ainsi que quelques textes de Fontaine qui accompagnent ses traductions: épîtres dédicatoires, avertissement du traducteur au lecteur, etc.

On ne peut donc que recommander la lecture de cet ouvrage à tous les curieux de la Renaissance, mais aussi à tous ceux qui aiment remettre en question la façon dont les manuels nous imposent ces hiérarchies entre minores et majores.

\section{AUTEURS \\ PASCALE CHIRON}

Université de Toulouse (UTM)

p.chirondelorme@wanadoo.fr 